\title{
The effect of soft contact lens thickness in visual function after intracorneal ring segments surgery
}

\author{
G. Carracedo*, J. Canales, P. Gonzalez, A. Recchioni, C. Carpena-Torres, J. Carballo-Álvarez \\ Department of Optics II (Optometry and Vision), School of Optics, Universidad Complutense de Madrid, Madrid, Spain
}

\section{A R T I C L E I N F O}

\section{Keywords:}

Keratoconus

Intracorneal rings

Visual function

Contact lenses

\begin{abstract}
A B S T R A C T
Objective: To study the influence of soft contact lens (SCL) central thickness and material in keratoconus on visual function after intracorneal ring segment (ICRS) surgery.

Methods: A pilot, experimental, prospective, cross-sectional and double-blind study was performed. Fourteen keratoconus patients with age range of $34.75 \pm 9.22$ years ( 7 males and 7 females) with ICRS implanted were involved in the study. Two different SCL materials [Hioxifilcon A (G-5X/p-GMA/HEMA) and Lucifilcon A (silicone-hydrogel)] with four different central thicknesses $(0.1,0.2,0.3$ and $0.4 \mathrm{~mm}$ ) were fitted in one eye per patient, selected randomly. High and low corrected distance visual acuity (CDVA) and contrast sensitivity (CS), corneal topography and corneal and total aberrometry were measured.

Results: Corneal spherical like, coma like and root mean square (RMS) decreased significantly for 0.3 and $0.4 \mathrm{~mm}$ in both SCL materials ( $\mathrm{p}<0.05$ ). Total RMS decreased significantly for $0.4 \mathrm{~mm}$ with both SCL materials $(\mathrm{p}<0.05)$. High and low CDVA improved for $0.4 \mathrm{~mm}$ of thickness for both materials $(\mathrm{p}<0.05)$. Statistically increasing were found in all thicknesses studied for CS in both materials ( $\mathrm{p}<0.05$ ).

Conclusion: A central thickness of the SCL equal or superior to $0.4 \mathrm{~mm}$ seems to decrease the ocular high order aberration (HOA) and to improve the visual function in keratoconus patients implanted with ICRS. However, the modulus of rigidity of the SCL would not influence the HOA correction.
\end{abstract}

\section{Introduction}

Keratoconus is the most prevalent corneal ectasia. It is characterized by a stromal thinning that causes a corneal protusion [1]. The optical result of this protusion is an increase in both ocular low-order aberrations (LOA), producing high values of myopic sphero-cylindrical errors, and high-order aberrations (HOA), mainly producing, however, abnormal values of coma aberration [2,3]. These HOA reduce visual function in keratoconic eyes below normative data [4]. Nowadays, there are two types of optical treatment: contact lenses fitting and intracorneal ring segment (ICRS) implantation.

ICRS implantation is a surgical procedure recommended to reestablish the corneal regularity [5]. ICRS are implanted in the corneal stroma in order to flatten the cone and to strengthen the cornea, resulting in a decrease of astigmatism and HOA which increases the visual function [6]. Despite the fact that the optical quality of the eye improves after the ICRS surgery [7], there is still a residue of HOA, mainly coma and spherical aberration, often due to partially decentered and superimposed ICRS on the pupil diameter respectively. This residual HOA reduces visual function in comparison with healthy eyes. In most cases after the surgery, the patients need to be compensated with contact lenses in order to decrease corneal HOA.

Rigid gas permeable (RGP) contact lenses fitting is the gold standard method for compensating HOA on ICRS implanted corneas [8]. RGP lenses create a tear meniscus between its posterior surface and anterior corneal surface that reestablishes the regularity of the optical anterior segment $[9,10]$. There are different designs in RGP materials, such as corneal [10], scleral [11], corneo-scleral [12] and hybrid contact lenses [13]. Despite HOA correction, patients occasionally report discomfort with corneal RGP $[14,15]$ and insertion difficulty with scleral lenses. These factors could induce intolerance to RGP lenses. Therefore, soft contact lenses (SCL) fitting is an option to improve comfort and visual satisfaction after ICRS implantation [16].

Apart from spectacles correction, conventional SCL with spherical and toric designs are usually fitted in incipient keratoconic eyes. However, in advanced stages or after ICRS implantation, the visual benefit of conventional SCL fitting is not widely reported [17-19]. Carballo-Álvarez et al. [19] found an improvement in visual function with spherical and toric SCL after ICRS implantation. High-contrast corrected distance visual acuity (CDVA) values in these groups were

\footnotetext{
* Corresponding author at: School of Optics, Department Optics II (Optometry and Vision), C/Arcos del Jalon 118, 28032 (Madrid), Spain.

E-mail address: gonzalocarracedo@gmail.com (G. Carracedo).
} 
similar to the piggy-back system (which includes both SCL and corneal RGP lenses) group. Furthermore, high thickness SCL is an alternative option in order to reestablish the symmetry of the optical anterior segment and decrease the HOA. In addition, high thickness SCL improves comfort during its wear and quality of life on irregular corneas [20-29]. Fernández-Velázquez and Fernández-Fidalgo [29] demonstrated an increase in high-contrast CDVA with high thickness SCL after ICRS implantation, these values being close to normative data.

The influence of SCL thickness in ocular HOA has never previously been described. For this reason, the purpose of this study was to study the effect of SCL central thickness and material on HOA correction in keratoconus after ICRS surgery. Furthermore, its influence on visual function was evaluated. The hypothesis was that central thickness and modulus of rigidity of SCL are directly proportional to HOA correction in keratoconus patients after ICRS surgery.

\section{Methods}

\subsection{Study design}

A pilot, experimental, prospective, cross-sectional and double-blind study was made. The study was conducted in compliance with good clinical practice guidelines, institutional review board regulations and following the tenets of the Declaration of Helsinki, reviewed and actualized in 2008 [30]. All participants were voluntarily included in the study after signing a written informed consent where complications associated to SCL wearing were explained. Participants were free to leave the study at any time. All the trials were performed in the Clinic of Optometry of the Faculty of Optics and Optometry (Complutense University of Madrid) by two different optometrists. The lenses were provided to the researchers with a coded label and without lens parameters data; only what measurement should be done with each lens. Then, neither the optometrist who perform the trials nor the patient knew the lens material and central thickness. Ocular wavefront aberrometry, corneal topography and visual function were measured before (PRE) and during different SCL wear.

\subsection{Sample}

Fourteen keratoconus patients ( 7 males and 7 females) with ICRS implanted were involved in the study. The measurements were performed in one eye per patient, selected randomly in the case that both were implanted with ICRS. The average age of participants was $34.75 \pm 9.22$ years, ranging from 19 to 48 years. The rest of their demographic characteristics are detailed in Table 1.

Inclusion criteria were keratoconus patients with Keraring ${ }^{\circ}$ (Mediphacos, Brazil) ICRS implanted $2.5 \mathrm{~mm}$ ( $5 \mathrm{~mm}$ of diameter) from corneal apex, understanding and signing the informed consent and knowing the indications and risks of wearing SCL. Exclusion criteria

Table 1

Demographic characteristics of participants in the study.

\begin{tabular}{ll}
\hline Parameter (mean (SD)) & Keratoconus \\
\hline Number of eyes (patients) & $14(14)$ \\
Age (years) & $34.75(9.22)$ \\
Age range (years) & {$[19.48]$} \\
Gender (male/female) & {$[7.7]$} \\
Sphere (D) & $-4.50(4.99)$ \\
Cylinder (D) & $-5.19(2.31)$ \\
High contrast BCVA (LogMAR) & $0.43(0.18)$ \\
Low contrast BCVA (LogMAR) & $0.69(0.20)$ \\
Contrast sensitivity (Log units) & $1.03(0.33)$ \\
Mean keratometry (D) & $50.52(3.34)$ \\
Flat K (D) & $48.54(3.58)$ \\
Steep K (D) & $52.49(3.05)$ \\
\hline
\end{tabular}

(p value $<0.05$. Wilcoxon for paired samples. For details see Methods.
Table 2

Characteristics of contact lenses used during the study.

\begin{tabular}{lll}
\hline Parameter & Contact lenses & \\
\hline Material (USAN) & Hioxifilcon A & Lucifilcon A \\
Material & p-GMA/HEMA & Silicone- Hydrogel \\
Dk (35 ${ }^{\circ}$ C. Fatt Units) & 21 & 60 \\
\% water content & 59 & 69 \\
Modulus index & 0.8 & 1.3 \\
Refractive index & 1.401 & 1.385 \\
Base curve (mm) & 7.10 to 8.90 (steps of 0.30) \\
Central Thickness (mm) & $0.1 ; 0.2 ; 0.3 ; 0.4$ \\
Diameter (mm) & 14.50 & \\
Power & Plano &
\end{tabular}

were any contraindication to wearing contact lens, any ocular pathology (except keratoconus), any other type of ICRS implanted and the use of systemic or ocular drugs that could affect the results.

\subsection{Soft contact lenses fitting}

Two SCL different materials [Hioxifilcon A (G-5X/p-GMA/HEMA) and Lucifilcon A (silicone-hydrogel)] with four different central thicknesses $(0.1,0.2,0.3$ and $0.4 \mathrm{~mm})$ and were provided by Lenticon Laboratories (Madrid, Spain). All contact lenses had a spherical design in both anterior and posterior surfaces. Their diameters were $14.50 \mathrm{~mm}$ and available base curve radii were 7.10 to $8.90 \mathrm{~mm}$ (steps of $0.30 \mathrm{~mm}$ ). The base curve fitted for lenses of 0.1 and $0.2 \mathrm{~mm}$ of central thickness was Flat $\mathrm{K}+0.3 \mathrm{~mm}$. The base curve fitted for lenses of 0.3 and 0.4 of central thickness was Flat $\mathrm{K}$, following manufacturer guidelines. Then, all patients wore the four central thickness for both contact lens materials. More technical details of contact lenses are shown in Table 2.

Before SCL fitting, the healthy state of the anterior surface was evaluated with a SL-D4 slit-lamp (Topcon, Tokyo, Japan). Ten minutes after lens insertion, once the tear secretion was normalized, the centration and movement were assessed. Then, measurements were performed. Two lenses were evaluated in each visit with $2 \mathrm{~h}$ of wash out period between lenses. In total, the patients were evaluated in four visits.

\subsection{Ocular wavefront aberrometry analysis}

All the aberrometric variables were expressed by Zernike polynomials according to the pyramidal criteria of the American National Standards Institute (ANSI) [31]. The analysis of ocular wavefront aberrometry was carried out from 3rd to 7th order. A $5 \mathrm{~mm}$ pupil diameter was chosen for the analysis, because the diameter of the ICRS implanted was $5 \mathrm{~mm}$. Before measuring, patients were in a room under mesopic conditions for ten minutes in order to obtain the largest pupil size as possible. The device L80 Wave + (Visionix, Chartres, France) was used for measuring the ocular, both total and corneal, wavefront aberrations. Total aberrations were measured three consecutive times with a Hartmann-Shack sensor, which uses near-infrared light of $780 \mathrm{~nm}$ wavelength. Corneal aberrations were measured with a Placido rings topography system. Both measurements were performed two seconds after blinking.

Aberrometric variables analyzed were spherical like, coma like and root means square (RMS) HOA. They were calculated by the following expressions:

SPHERICAL LIKE $=\sqrt{\left(Z_{12}\right)^{2}+\left(Z_{24}\right)^{2}}$

Where $\mathrm{Z}_{12}$ and $\mathrm{Z}_{24}$ are the Zernike coefficients of spherical aberration of 4 th and 6 th order respectively.

COMA LIKE $=\sqrt{\left(Z_{7}\right)^{2}+\left(Z_{8}\right)^{2}+\left(Z_{17}\right)^{2}+\left(Z_{18}\right)^{2}+\left(Z_{31}\right)^{2}+\left(Z_{32}\right)^{2}}$ 
Table 3

Total and corneal high order aberrations (HOA) for different thicknesses and materials.

\begin{tabular}{|c|c|c|c|c|c|}
\hline Parameter & CL Thickness & $5 \mathrm{GX}$ & $P$ Value & $\mathrm{HiSi}$ & $\mathrm{P}$ value \\
\hline \multirow[t]{5}{*}{ Corneal spherical like $(\mu \mathrm{m})$ (mean (SD)) } & Pre & $0.975(0.557)$ & - & $0,975(0,557)$ & - \\
\hline & 0.1 & $0.638(0.512)$ & 0.551 & $0.656(0.564)$ & 0.133 \\
\hline & 0.2 & $0.636(0.538)$ & 0.087 & $0.523(0.500)$ & 0.198 \\
\hline & 0.3 & $0.513(0.429)$ & 0.363 & $0.484(0.434)$ & $0.030^{*}$ \\
\hline & 0.4 & $0.434(0.257)$ & $0.041^{*}$ & $0.399(0.347)$ & $0.013^{*}$ \\
\hline \multirow[t]{5}{*}{ Corneal coma like $(\mu \mathrm{m})$ (mean (SD)) } & Pre & $2.733(1.348)$ & - & $2.733(1.348)$ & - \\
\hline & 0.1 & $2.725(1.345)$ & 0.221 & $2.36(1.108)$ & $0.007 * *$ \\
\hline & 0.2 & $2.582(1.328)$ & 0.140 & $2.330(1.071)$ & $0.001 * *$ \\
\hline & 0.3 & $2.136(1.119)$ & $0.047^{*}$ & $2.327(1.143)$ & $0.001^{* *}$ \\
\hline & 0.4 & $1.812(1.033)$ & $0.035^{*}$ & $1.812(1.011)$ & $0.001 * *$ \\
\hline \multirow[t]{5}{*}{ Corneal RMS $(\mu \mathrm{m})$ (mean (SD)) } & Pre & $3.375(2.220)$ & - & $3.375(2.220)$ & - \\
\hline & 0.1 & $3.141(1.431)$ & 0.064 & $2.752(1.288)$ & $0.018^{*}$ \\
\hline & 0.2 & $2.958(1.272)$ & 0.096 & $2.695(1.313)$ & $0.002^{* *}$ \\
\hline & 0.3 & $2.612(1.271)$ & $0.05^{*}$ & $2.632(1.267)$ & $0.001 * *$ \\
\hline & 0.4 & $2.257(1.086)$ & $0.035^{*}$ & $2.235(1.239)$ & $0.001^{* *}$ \\
\hline \multirow[t]{5}{*}{ Total spherical like $(\mu \mathrm{m})$ (mean (SD)) } & Pre & $0.285(0.203)$ & - & $0.285(0.203)$ & - \\
\hline & 0.1 & $0.492(0.223)$ & $0.004 * *$ & $0.564(0.258)$ & $<0.001 *$ \\
\hline & 0.2 & $0.609(0.293)$ & $<0.001^{* *}$ & $0.564(0.244)$ & $0.003 * *$ \\
\hline & 0.3 & $0.438(0.295)$ & $0.008^{* *}$ & $0.323(0.258)$ & 0.534 \\
\hline & 0.4 & $0.321(0.224)$ & 0.143 & $0.345(0.244)$ & 0.751 \\
\hline \multirow[t]{5}{*}{ Total coma like $(\mu \mathrm{m})($ mean $(\mathrm{SD}))$} & Pre & $1.277(0.928)$ & - & $1.277(0.928)$ & - \\
\hline & 0.1 & $1.325(0.843)$ & 0.802 & $1.682(0.959)$ & 0.061 \\
\hline & 0.2 & $1.715(0.983)$ & 0.101 & $1.484(1.022)$ & 0.058 \\
\hline & 0.3 & $1.201(0.682)$ & 0.750 & $1.140(0.751)$ & 0.909 \\
\hline & 0.4 & $0.878(0.611)$ & $0.012^{*}$ & $0.978(0.720)$ & 0.218 \\
\hline \multirow[t]{5}{*}{ Total RMS $(\mu \mathrm{m})$ (mean (SD)) } & Pre & $1.597(0.971)$ & - & $1.597(0.971)$ & - \\
\hline & 0.1 & $1.771(1.127)$ & 0.524 & $2.067(1.182)$ & 0.128 \\
\hline & 0.2 & $2.130(0.995)$ & 0.068 & $1.863(1.086)$ & 0.082 \\
\hline & 0.3 & $1.556(0.719)$ & 0.699 & $1.499(0.758)$ & 0.713 \\
\hline & 0.4 & $1.229(0.671)$ & $0.024^{*}$ & $1.293(0.718)$ & $0.038^{*}$ \\
\hline
\end{tabular}

*p $<0.05$. Comparison post-wear with pre-wear values; Wilcoxon test

$* * \mathrm{p}<0.01$.

Where $Z_{7}, Z_{17}$ and $Z_{31}$ are the Zernike coefficients of vertical coma aberration and $\mathrm{Z}_{8}, \mathrm{Z}_{18}$ and $\mathrm{Z}_{32}$ of horizontal coma aberration of 3rd, 5th and 7 th order respectively.

RMS HOA $=\sum_{i=6}^{i=35} \sqrt{\left(Z_{i}\right)^{2}}$

Where $Z_{i}$ are the Zernike coefficients from 3rd to 7 th order.

\subsection{Visual function}

Corrected distance visual acuity (CDVA) and contrast sensitivity (CS) were measured with the best spectacle correction under photopic conditions $\left(85 \mathrm{~cd} / \mathrm{m}^{2}\right)$ and physiological pupil sizes. All the parameters of visual function were analyzed with logMAR units in order to do the statistical analysis. CDVA was measured using the Bailey-Lovie highcontrast (96\%) and low-contrast (10\%) letter charts (Precision Vision, LaSalle, Illinois, USA) at $4 \mathrm{~m}$. CS was measured using the Pelli-Robson chart (Precision Vision, LaSalle, Illinois, USA) at $1 \mathrm{~m}$, which spatial frequency corresponds to 1 cycle per degree (cpd). To simulate the lenses available in the market and taking in account that the majority of manufacturers declare a mean central thickness of $0.40 \mathrm{~mm}$, the measurements of visual function were only performed with the extreme central thicknesses $(0.1$ and $0.4 \mathrm{~mm})$.

\subsection{Statistical analysis}

Statistical analysis was performed using SPSS Statistics 22 software (IBM, Chicago, Illinois, USA). The sample size calculation was performed with statistical software (Granmo 6.0; Institut Municipal d'Investigación Medica, Barcelona, Spain). With an accepted two-sided statistical significance threshold of 0.05 and a risk of 0.20 , for a standard deviation of 1.2 units for the main variable (HOA RMS) and in order to detect a difference of 1.0 units or more, 14 subjects were needed to find statistically significant differences. It has been anticipated a drop-out rate of $15 \%$. The normal distribution of the variables was assessed using the Saphiro-Wilk test, resulting in non-normality because of the heterogeneity of the keratoconus patients after ICRS surgery. According to these results, the Wilcoxon signed-rank test was chosen in order to compare the values of ocular wavefront aberrometry, corneal topography, subjective refraction and visual function before (PRE) and after fitting the different SCL. A statistical significance of $95 \%$ was established $(\mathrm{p}<0.05)$. The results are shown as mean $\pm \mathrm{SD}$

\section{Results}

\subsection{Ocular wavefront aberrometry}

All aberrometric data are summarized in Table 3. The results of corneal wavefront aberrations are shown in Fig. 1. With the lenses of G5X material, there was a statistically significant decrease in the corneal spherical like for $0.4 \mathrm{~mm}(\mathrm{p}=0.041)$ and in both coma like ( $\mathrm{p}<0.01$ ) and RMS HOA ( $\mathrm{p}<0.05$ and $\mathrm{p}<0.01$ ) for 0.3 and $0.4 \mathrm{~mm}$. With the lenses of silicone-hydrogel material, there was a statistically significant decrease in corneal spherical like for 0.3 and $0.4 \mathrm{~mm}$ and coma like and RMS HOA for all the central thicknesses $(\mathrm{p}<0.05)$. The results obtained without statistical differences were also lower than PRE condition. Corneal HOA correction was directly proportional to central thickness with both SCL materials Fig. 1.

Fig. 2 shows the results of total wavefront aberrations. With the lenses of G-5X material, there was a statistically significant increase in total spherical like for $0.1,0.2$ and $0.3 \mathrm{~mm}(\mathrm{p}<0.01)$. In contrast, 
A

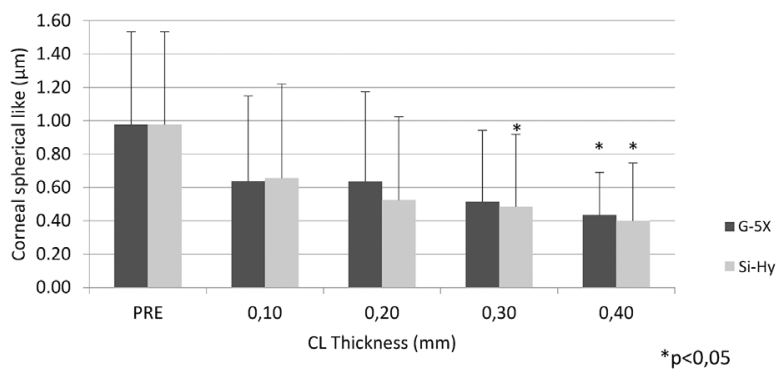

B

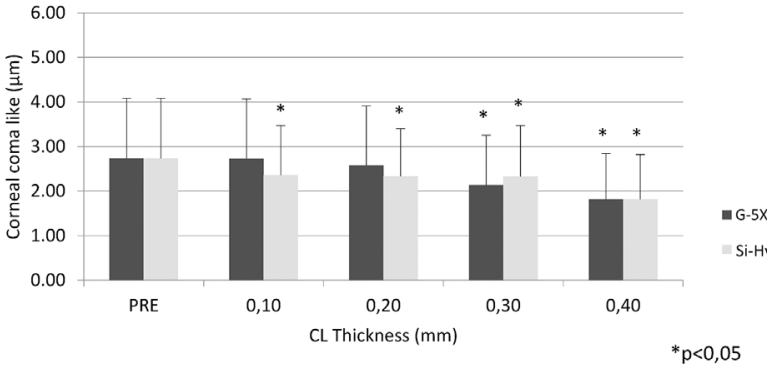

C

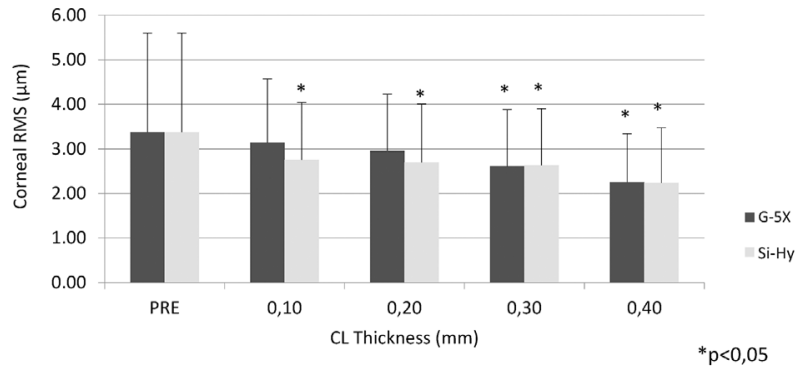

Fig. 1. Bar graph representing the trend of corneal HOA in function of contact lens thickness for hydrophilic and hydrogel silicone materials. ${ }^{*} \mathrm{p}<0.05$. Comparison postwear with pre-wear values; Wilcoxon test.

there was a statistically significant decrease in total coma like and RMS HOA for $0.4 \mathrm{~mm}(\mathrm{p}<0.05)$. With the lenses of silicone-hydrogel material, there was a statistically significant increase in spherical like for 0.1 and $0.2 \mathrm{~mm}(\mathrm{p}<0.01)$ and a statistically significant decrease in RMS HOA of $0.4 \mathrm{~mm}(\mathrm{p}=0.038)$.

\subsection{Corneal topography and subjective refraction}

Corneal topography and subjective refraction data, with and without SCL, are summarized in Table 4 . There was a statistically significant decrease $(\mathrm{p}<0.05$ and $\mathrm{p}<0.01$ ) in flat $\mathrm{K}$, steep $\mathrm{K}$ and Kmean (D) for all the SCL. The flattening of the anterior surface of the SCL was directly proportional to their central thicknesses. Regarding sphero-cylindrical values, there was a statistically significant decrease in sphere with the lenses of G-5X material for $0.3(\mathrm{p}<0.05)$ and $0.4 \mathrm{~mm}(\mathrm{p}<0.01)$ and with the lenses of silicone-hydrogel for all the central thicknesses $(\mathrm{p}<0.05)$.

\subsection{Visual function}

Visual function results are shown in Fig. 3. The mean, high-contrast CDVA (logMAR) was $0.43 \pm 0.18$ for PRE condition. With the lenses of G-5X material, the mean was $0.41 \pm 0.21$ for $0.1 \mathrm{~mm}(\mathrm{p}=0.115)$ and

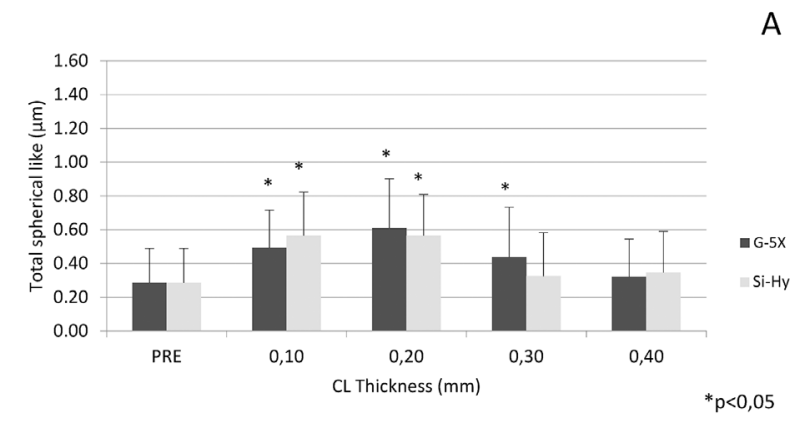

B

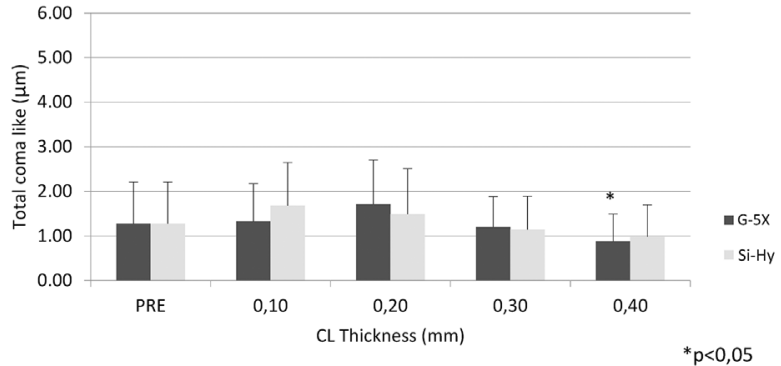

C

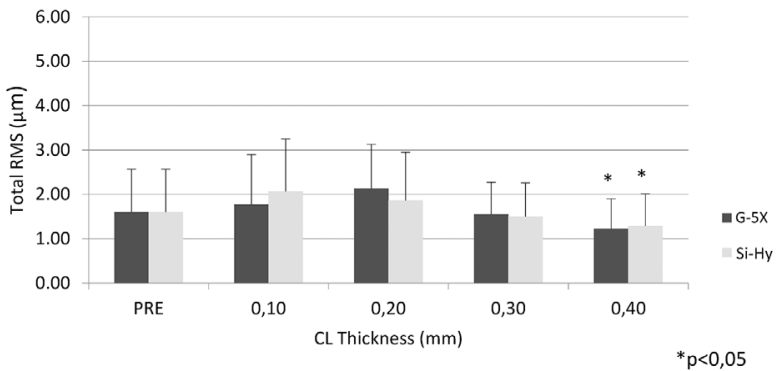

Fig. 2. Bar graph representing the trend of total HOA in function of contact lens thickness for hydrophilic and hydrogel silicone materials. ${ }^{*} \mathrm{p}<0.05$. Comparison post-wear with pre-wear values; Wilcoxon test.

$0.28 \pm 0.13$ for $0.4 \mathrm{~mm}(\mathrm{p}=0.046)$. With the lenses of silicone-hydrogel, the mean was $0.37 \pm 0.19$ for $0.1 \mathrm{~mm}(\mathrm{p}=0.345)$ and $0.29 \pm 0.18$ for $0.4 \mathrm{~mm}(\mathrm{p}=0.043)$. In addition, there was a statistically significant improvement $(\mathrm{p}=0.044)$ in high-contrast CDVA with $0.4 \mathrm{~mm}$ respect to $0.1 \mathrm{~mm}$ in G-5X material. The same pattern was observed for silicone-hydrogel material, despite there being no significant differences.

The mean low-contrast CDVA (logMAR) was $0.69 \pm 0.20$ for PRE condition. With the lenses of G-5X material, the mean was $0.67 \pm 0.18$ for $0.1 \mathrm{~mm}(\mathrm{p}=0.249)$ and $0.55 \pm 0.17(\mathrm{p}=0.028)$. With the lenses of silicone-hydrogel material, the mean was $0.60 \pm 0.17$ for $0.1 \mathrm{~mm}$ $(\mathrm{p}=0.116)$ and $0.52 \pm 0.23$ for $0.4 \mathrm{~mm}(\mathrm{p}=0.046)$. Comparing the two central thicknesses, there was a significant improvement in lowcontrast CDVA with $0.4 \mathrm{~mm}$ for both $\mathrm{G}-5 \mathrm{X}(\mathrm{p}=0.024)$ and siliconehydrogel $(\mathrm{p}=0.046)$.

The mean CS (logMAR) was $1.03 \pm 0.33$ for PRE condition. With the lenses of G-5X material, the mean was $1.10 \pm 0.40$ for $0.1 \mathrm{~mm}$ ( $\mathrm{p}=0.043$ ) and $1.23 \pm 0.29$ for $0.4 \mathrm{~mm}(\mathrm{p}=0.043)$. With the lenses of silicone-hydrogel material, the mean was $1.13 \pm 0.29$ for $0.1 \mathrm{~mm}$ ( $\mathrm{p}=0.044)$ and $1.23 \pm 0.40$ for $0.4 \mathrm{~mm}(\mathrm{p}=0.043)$. There were no statistically significant differences $(p>0.05)$ in CS between extreme central thicknesses for both materials. 
Table 4

Keratometry and refraction values for different thicknesses and materials.

\begin{tabular}{|c|c|c|c|c|c|}
\hline Parameter & CL Thickness & $5 \mathrm{GX}$ & P Value & $\mathrm{HiSi}$ & $P$ value \\
\hline \multirow[t]{5}{*}{ Flat K (D) (mean (SD)) } & Pre & $48.54(3.58)$ & - & $48.54(3.58)$ & - \\
\hline & 0.1 & $45.84(5.92)$ & $0.005^{* *}$ & $46.16(4.75)$ & $0.009 *$ \\
\hline & 0.2 & $45.05(6.03)$ & $0.001 * *$ & $45.59(5.23)$ & $0.002^{*}$ \\
\hline & 0.3 & 44.34 (6.09) & $0.002^{* *}$ & $44.93(5.41)$ & $0.001 *$ \\
\hline & 0.4 & $44.69(6.03)$ & $0.023^{*}$ & 44.28 (5.39) & $0.001 *$ \\
\hline \multirow[t]{5}{*}{ Steep K (D) (mean (SD)) } & Pre & $52.49(3.05)$ & -- & $52.49(3.05)$ & -- \\
\hline & 0.1 & $49.91(5.90)$ & $0.003^{* *}$ & $50.51(4.72)$ & $0.002^{*}$ \\
\hline & 0.2 & $49.18(6.04)$ & $0.001^{* *}$ & $49.18(4.80)$ & $0.001 *$ \\
\hline & 0.3 & $47.39(5.11)$ & $0.001^{* *}$ & $48.55(5.48)$ & $0.001 *$ \\
\hline & 0.4 & $47.59(5.53)$ & $0.011^{*}$ & $47.39(5.10)$ & $0.001 *$ \\
\hline \multirow[t]{5}{*}{ Kmean (D) (mean (SD)) } & Pre & $50.52(3.34)$ & - & $50.52(3.34)$ & - \\
\hline & 0.1 & $47.79(6.12)$ & $0.003^{* *}$ & $48.27(4.91)$ & $0.002^{*}$ \\
\hline & 0.2 & $46.99(6.24)$ & $0.001^{* *}$ & $47.12(5.16)$ & $0.001 *$ \\
\hline & 0.3 & $45.78(5.64)$ & $0.001^{* *}$ & $46.67(5.63)$ & $0.001 *$ \\
\hline & 0.4 & $46.09(5.84)$ & $0.023^{*}$ & $45.78(5.36)$ & $0.001 *$ \\
\hline \multirow[t]{5}{*}{ Sphere (D) (mean (SD)) } & Pre & $-4.50(4.99)$ & - & $-4.50(4.99)$ & - \\
\hline & 0.1 & $-1.68(4.70)$ & 0.094 & $-3.36(5.30)$ & $0.036^{*}$ \\
\hline & 0.2 & $-2.67(4.70)$ & 0.087 & $-3.00(5.34)$ & $0.034 *$ \\
\hline & 0.3 & $-2.17(4.56)$ & $0.023^{*}$ & $-2.73(4.59)$ & $0.010^{*}$ \\
\hline & 0.4 & $-2.04(3.52)$ & $0.008 * *$ & $-2.47(4.40)$ & $0.011^{*}$ \\
\hline \multirow[t]{5}{*}{ Cylinder (D) (mean (SD)) } & Pre & $-5.19(2.31)$ & - & $-5.19(2.31)$ & - \\
\hline & 0.1 & $-4.23(3.84)$ & $0.041^{*}$ & $-4.31(3.17)$ & $0.021 *$ \\
\hline & 0.2 & $-4.07(3.23)$ & $0.012^{*}$ & $-4.39(3.56)$ & 0.057 \\
\hline & 0.3 & $-3.56(3.00)$ & $0.002^{* *}$ & $-3.67(3.10)$ & $0.008^{*}$ \\
\hline & 0.4 & $-3.15(2.48)$ & $0.002^{* *}$ & $-3.67(2.97)$ & $0.002 *$ \\
\hline
\end{tabular}

${ }^{*} \mathrm{p}<0.05$. Comparison post-wear with pre-wear values; Wilcoxon test.

$* * \mathrm{p}<0.01$.

\section{Discussion}

In recent years, SCL fitting has proved to be an alternative to RGP lenses on irregular corneas, including ICRS implanted, in order to improve optical and visual quality [17-29]. SCL are especially recommended in patients who want to increase their comfort, assuming a reduction of visual function compared to RGP wearers [16]. Lathed-SCL materials have lower values of oxygen permeability than RGP materials [32]. Therefore, oxygen transmissibility to the cornea is limited with high thickness SCL, and, even in the case of lenses with normal thickness, the power of the lens also changes significantly the central and peripheral thicknesses having a significant impact on the oxygen transmissibility. Then, a reduction in contact lenses continuous wearing time is often necessary [33]. Corneal hypoxia and edema are risks associated to high thickness SCL in abusive wearers.

Corneal and total HOA, especially coma and spherical aberrations, are still uncorrected in keratoconus patients after ICRS surgery as shown in the results of the present study for PRE condition and other studies [7], which affects visual function. HOA reduction during SCL wearing has been demonstrated in keratoconus patients before ICRS implantation. Jinabhai et al. $[17,18]$ found a correction of between $30 \%$ and $40 \%$ approximately of total RMS HOA with conventional toric SCL in keratoconic eyes, mainly associated to a reduction of coma aberration. There is no other study that shows the influence of conventional SCL in HOA of keratoconus patients. Gumus and Kahraman [25] found a decrease in total RMS HOA between 15\% and 33\% in keratoconus patients fitted with high thickness SCL which central thicknesses had a range of $0.35-0.59 \mathrm{~mm}$. Conventional SCL, high thickness SCL and RGP lenses compensate the anterior corneal HOA in keratoconic eyes. However, a residual value of posterior HOA still exists, which affects the optical and visual quality [34]. With the aim of correcting the total HOA in keratoconic eyes with SCL, different studies designed customized SCL using wavefront-guided technology [18,35-39] or incorporating differences in superior and inferior thicknesses [40]. These results showed a HOA correction similar to RGP lenses. Despite this HOA correction, López-Gil et al. [35] and Jinabhai et al. [18] found a reduction in the visual function compared to normal eyes and RGP keratoconic wearers respectively. In contrast, Sabesan et al. [37] and Marsack et al. [38,39] found an improvement in high and low contrast CDVA compared to conventional SCL (the first authors) and RGP keratoconic wearers (both authors). In addition, Katsoulos et al. [40] found an improvement in high and low contrast CDVA compared to spectacles correction in these keratoconus patients.

In the present study, a decrease in corneal HOA, directly proportional to the central thickness of the SCL (Table 3) has been found. With the G-5X material lenses, the statistical reduction in RMS HOA $22.61 \%$ for $0.3 \mathrm{~mm}$ and $33.13 \%$ for $0.4 \mathrm{~mm}$. With silicone-hydrogel lenses, the statistical reduction in RMS HOA was $22.02 \%$ for $0.3 \mathrm{~mm}$ and $33.88 \%$ for $0.4 \mathrm{~mm}$. These results were associated to a reduction in corneal coma aberration, mainly, and spherical aberration. In addition, the flattening of the anterior surface Kmean was also directly proportional to the central thickness of the SCL (Table 4). Therefore, it can be deduced that an increase in the central thickness of the SCL maintains the shape of these lenses on the ocular surface, reducing the corneal HOA. Furthermore, no statistical differences between G-5X and silicone-hydrogel SCL were found, indicating that the modulus of rigidity of these lenses would not influence corneal HOA correction.

The pattern of the total HOA measurements does not correspond to the corneal HOA values. There was an increase in total spherical like for all the central thicknesses with both lenses. In total coma like, there was only a statistically significant decrease for the lenses of G-5X material and $0.4 \mathrm{~mm}$ of central thickness. In addition, in total RMS HOA there was only a decrease in the central thicknesses of $0.4 \mathrm{~mm}$ in both materials (18.66\% for G-5X and $19.04 \%$ for silicone-hydrogel), lower than corneal RMS HOA. These differences between corneal and total HOA values could be explained by different hypothesis which are shown below.

The corneal HOA measurements with the SCL fitted are taken over the anterior surface of these lenses. Therefore, the precorneal tear meniscus, the posterior corneal surface, which is aberrated in keratoconus patients [34], and the internal structures does not affect corneal HOA. Another factor is the influence of the ICRS implanted $2.5 \mathrm{~mm}$ 

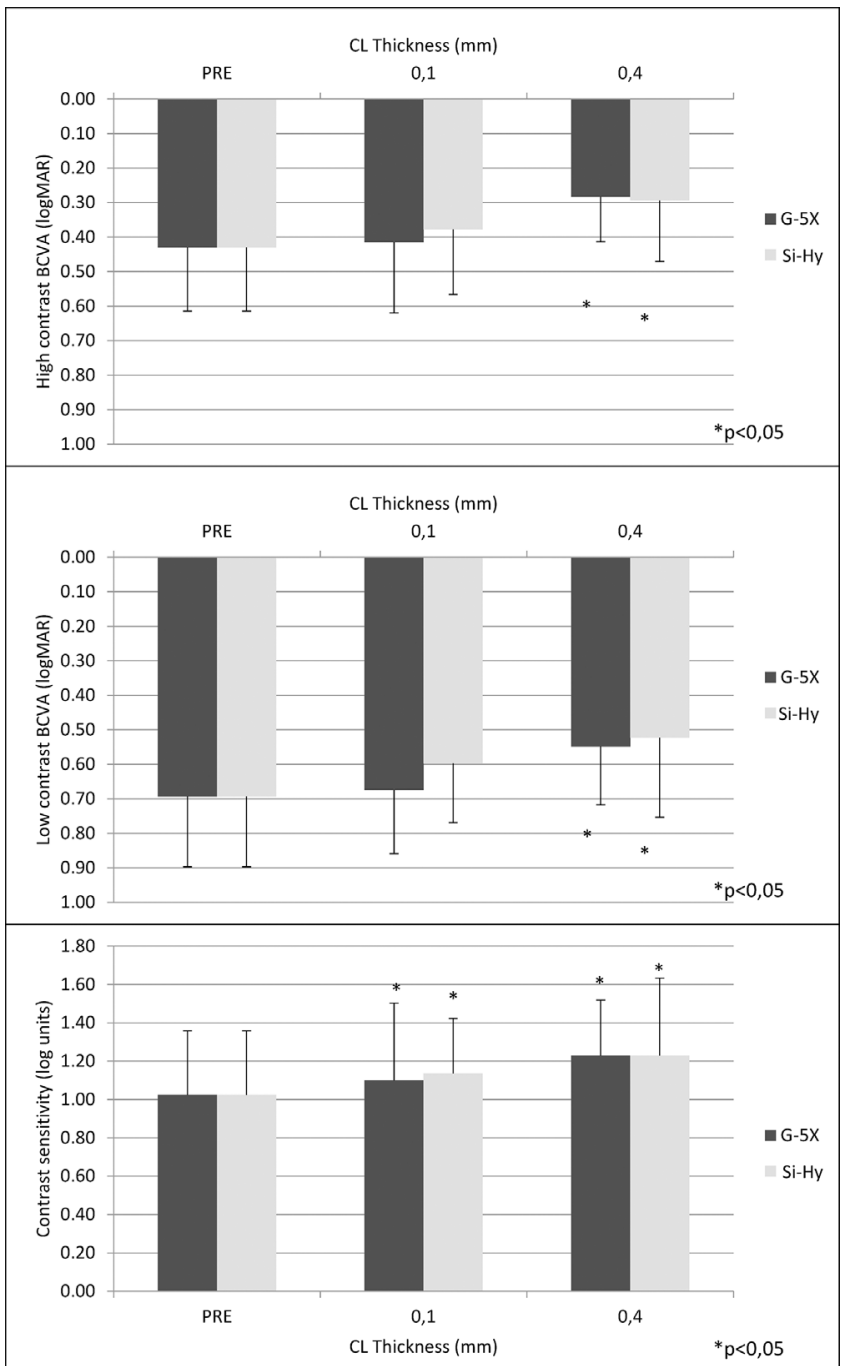

Fig. 3. Visual function parameters comparison between the lowest and highest contact lens thickness studied in both materials. ${ }^{*} \mathrm{p}<0.05$. Comparison post-wear with prewear values; Wilcoxon test.

( $5 \mathrm{~mm}$ of diameter) from the corneal apex. In concordance, the HOA measurements were taken with a pupil size of $5 \mathrm{~mm}$, so ICRS implanted would mainly affect total HOA. In addition, the loss of water content in the material (present in different percentages in G-5X and silicone-hydrogel materials) could have generated scattering phenomena [41], affecting the optical quality of the anterior surface of the SCL and the eye. Tear film quality disorders reported in keratoconus patients could also affect total and corneal HOA measurements [42,43], added to the reduction in the repeatability of the corneal [44] and total [45] HOA in keratoconic eyes. Finally, the decentering of the SCL after blinking could have induced coma and spherical aberrations.

High thickness SCL has been shown as an alternative to RGP lenses to correct the HOA in order to improve the visual function in irregular corneas, including ICRS implanted keratoconic eyes. Carballo-Álvarez et al. [19] found an increase in high-contrast CDVA of 0.07 logMAR and $0.14 \log$ MAR in keratoconus patients with ICRS after fitting conventional toric SCL and spherical SCL respectively. The only scientific evidence of the influence of high thickness SCL in visual function in keratoconus patients after ICRS implantation was a study of FernándezVelázquez and Fernández-Fidalgo [29]. The authors found an increase in high-contrast CDVA between 0.17 logMAR and 0.30 logMAR after fitting different designs of SCL with central thicknesses superior than $0.4 \mathrm{~mm}$. In accordance, the results of the present study shows a statistical increase in high-contrast CDVA with the SCL of $0.4 \mathrm{~mm}$ of central thickness of $0.15 \log$ MAR for G-5X material and 0.14 for silicone-hydrogel material. In low-contrast CDVA, a statistical increase with the lenses of $0.4 \mathrm{~mm}$ central thickness of $0.14 \operatorname{logMAR}$ and 0.17 logMAR for G-5X and silicone-hydrogel materials respectively was also found. Finally, in CS a statistical increase of $0.20 \log$ MAR with the SCL of $0.4 \mathrm{~mm}$ for both materials was found. These results of the visual function are consistent with the results of the total RMS HOA correction, which show a statistically significant decrease of the SCL of $0.4 \mathrm{~mm}$ of central thickness for both materials. Again, it becomes clear that the central thickness is the parameter of the SCL, which improves HOA correction and visual function instead of the modulus of rigidity.

The present study had some limitations that could be improved upon in future studies. It would be necessary: to increase the sample size, to analyze the wavefront aberrometry and to measure the visual function under photopic and mesopic conditions, to analyze the influence of the base curve of the SCL in the centration and movement because it influences the aberrometric values and to increase the wearing time of the SCL before measuring.

In conclusion, a central thickness of the SCL equal or superior to $0.4 \mathrm{~mm}$ seems to decrease the ocular HOA and to improve the visual function in keratoconus patients implanted with ICRS. However, the modulus of rigidity of the SCL does not influence the HOA correction. Taking in account the clinical safety by the loss of oxygen transmissibility, high thickness SCL fitting could be suggested as an alternative to RGP lenses to partially compensate the residual HOA after ICRS implantation in order to improve the visual function and the comfort. Therefore, the choice of the high thickness SCL materials should be prioritized mainly by their oxygen permeability following by the comfort.

\section{Disclosure}

The authors do not have any financial interest on the materials and instruments used in this study

\section{Acknowledgements}

This research was funded by the Spanish Ministry of Economy and Competition (Project SAF2013-44416R and SAF2016-77084R) and the Ministry of Health Social Services and Equality (Project RETICS RD12/ 0034/0003).

\section{References}

[1] M. Romero-Jimenez, J. Santodomingo-Rubido, J.S. Wolffsohn, Keratoconus: a review, Cont. Lens Anterior Eye 33 (2010) 157-166.

[2] R. Kosaki, N. Maeda, K. Bessho, Y. Hori, K. Nishida, A. Suzaki, Y. Hirohara T. Mihashi, T. Fujikado, Y. Tano, Magnitude and orientation of zernike terms in patients with keratoconus, Invest. Ophthalmol. Vis. Sci. 48 (2007) 3062-3068.

[3] D.P. Pinero, J.L. Alio, A. Aleson, M. Escaf, M. Miranda, Pentacam posterior and anterior corneal aberrations in normal and keratoconic eyes, Clin. Exp. Optom. 92 (2009) 297-303.

[4] W.B. Khor, R.H. Wei, L. Lim, C.M. Chan, D.T. Tan, Keratoconus in Asians: demographics: clinical characteristics and visual function in a hospital-based population, Clin. Exp. Ophthalmol. 39 (2011) 299-307.

[5] A. Vega-Estrada, J.L. Alio, The use of intracorneal ring segments in keratoconus, Eye Vis. (Lond.) 3 (2016) 8.

[6] J. Carballo, M.C. Puell, R. Cuina, J.M. Vazquez, J.M. Benitez-del-Castillo, Changes in visual function under mesopic and photopic conditions after intrastromal corneal ring segment implantation for different stages of keratoconus, J. Cataract Refract. Surg. 39 (2013) 393-402.

[7] A. Vega-Estrada, J.L. Alio, L.F. Brenner, N. Burguera, Outcomes of intrastromal corneal ring segments for treatment of keratoconus: five-year follow-up analysis, $\mathrm{J}$ Cataract Refract. Surg. 39 (2013) 1234-1240.

[8] L.E. Downie, Lindsay RG: Contact lens management of keratoconus, Clin. Exp. Optom. 98 (2015) 299-311.

[9] J.D. Marsack, K.E. Parker, K. Pesudovs, W.J. Donnelly III, R.A. Applegate, Uncorrected wavefront error and visual performance during RGP wear in keratoconus, Optom. Vis. Sci. 84 (2007) 463-470.

[10] K. Negishi, T. Kumanomido, Y. Utsumi, K. Tsubota, Effect of higher-order aberrations on visual function in keratoconic eyes with a rigid gas permeable contact lens, 


\section{Am. J. Ophthalmol. 144 (2007) 924-929.}

[11] G. Carracedo, M. Serramito-Blanco, A. Martin-Gil, Z. Wang, C. Rodriguez-Pomar, J. Pintor, Post-lens tear turbidity and visual quality after scleral lens wear, Clin. Exp. Optom. (2017) (Epub ahead of print).

[12] E. van der Worp, D. Bornman, D.L. Ferreira, M. Faria-Ribeiro, N. Garcia-Porta, J.M. Gonzalez-Meijome, Modern scleral contact lenses: a review, Cont. Lens Anterior Eye 37 (2014) 240-250.

[13] H. Hashemi, N. Shaygan, S. Asgari, F. Rezvan, S. Asgari, ClearKone-Synergeyes or rigid gas-Permeable contact lens in keratoconic patients: a clinical decision, Eye Contact Lens 40 (2014) 95-98.

[14] T.B. Edrington, R.E. Gundel, D.P. Libassi, H. Wagner, G.E. Pierce, J.J. Walline, J.T. Barr, H.E. Olafsson, K. Steger-May, J. Achtenberg, B.S. Wilson, M.O. Gordon, $\mathrm{K}$. Zadnik, Variables affecting rigid contact lens comfort in the collaborative longitudinal evaluation of keratoconus (CLEK) study, Optom. Vis. Sci. 81 (2004) 182-188.

[15] G. Carracedo, A. Martin-Gil, S.C. Peixoto-de-Matos, P. Abejon-Gil, R. Macedo-deAraujo, J.M. Gonzalez-Meijome, Symptoms and signs in rigid gas permeable lens wearers during adaptation period, Eye Contact Lens 42 (2016) 108-114.

[16] E.H. Yildiz, M. Erdurmus, E.S. Elibol, B. Acar, E.T. Vural, Contact lens impact on quality of life in keratoconus patients: rigid gas permeable versus soft silicone-hydrogel keratoconus lenses, Int. J. Ophthalmol. 8 (2015) 1074-1077.

[17] A. Jinabhai, H. Radhakrishnan, C. Tromans, C. O'Donnell, Visual performance and optical quality with soft lenses in keratoconus patients, Ophthalmic Physiol. Opt. 32 (2012) 100-116.

[18] A. Jinabhai, C. O'Donnell, C. Tromans, H. Radhakrishnan, Optical quality and visual performance with customised soft contact lenses for keratoconus, Ophthalmic Physiol. Opt. 34 (2014) 528-539.

[19] J. Carballo-Alvarez, M.C. Puell, R. Cuiña, D. Diaz-Valle, J.M. Vazquez, J.M. BenitezDel-Castillo, Soft contact lens fitting after intrastromal corneal ring segment implantation to treat keratoconus, Cont. Lens Anterior Eye 37 (2014) 377-381.

[20] J.M. Gonzalez-Meijome, J. Jorge, J.B. de Almeida, M.A. Parafita, Soft contact lenses for keratoconus: case report, Eye Contact Lens 32 (2006) 143-147.

[21] F.J. Fernandez-Velazquez, Kerasoft IC compared to Rose-K in the management of corneal ectasias, Cont. Lens Anterior Eye 35 (2012) 175-179.

[22] O.O. Ucakhan, B. Bayraktutar, KeraSoft 3 contact lenses in corneal ectasia, Eye Contact Lens 40 (2014) 390-394.

[23] S. Su, L. Johns, M.J. Rah, R. Ryan, J. Barr, Clinical performance of KeraSoft((R)) IC in irregular corneas, Clin. Ophthalmol. 9 (2015) 1953-1964.

[24] A.L. Lagina, Soft contact lens optimizes visual goals for a patient with keratoectasia, Optom. Vis. Sci. (2015) 92

[25] K. Gumus, N. Kahraman, A new fitting approach for providing adequate comfort and visual performance in keratoconus: soft HydroCone (Toris K) lenses, Eye Contact Lens 42 (2016) 225-230.

[26] P. Sultan, C. Dogan, G. Iskeleli, A retrospective analysis of vision correction and safety in keratoconus patients wearing Toris K soft contact lenses, Int. Ophthalmol. 6 (2016) 799-805.

[27] I. Yilmaz, F. Ozcelik, B. Basarir, G. Demir, G. Durusoy, M. Taskapili, Clinical performance of toris $\mathrm{K}$ contact lens in patients with moderate to advanced keratoconus: a real life retrospective analysis, J. Ophthalmol. (2016) 2358901.
[28] D. Ozek, O.E. Kemer, N. Bayraktar, Hydro cone lens visual performance and impact on quality of life in irregular corneas, Cont. Lens Anterior Eye 39 (2016) 458-460.

[29] F.J. Fernandez-Velazquez, M.J. Fernandez-Fidalgo, Feasibility of custom-made hydrogel contact lenses in keratoconus with previous implantation of intracorneal ring segments, Cont. Lens Anterior Eye 38 (2015) 351-356.

[30] K.S. Puri, K.R. Suresh, N.J. Gogtay, U.M. Thatte, Declaration of Helsinki: 2008: implications for stakeholders in research, J. Postorad. Med. 55 (2009) 131-134.

[31] C. McAlinden, M. McCartney, J. Moore, Mathematics of Zernike polynomials: a review, Clin. Experiment. Ophthalmol. 39 (2011) 820-827.

[32] H. Ichijima, H.D. Cavanagh, How rigid gas-permeable lenses supply more oxygen to the cornea than silicone hydrogels: a new model, Eye Contact Lens 33 (2007) 216-223.

[33] M. Lira, C. Pereira, M.E. Real Oliveira, E.M. Castanheira, Importance of contact lens power and thickness in oxygen transmissibility, Cont. Lens Anterior Eye 38 (2015) $120-126$.

[34] M. Chen, G. Yoon, Posterior corneal aberrations and their compensation effects on anterior corneal aberrations in keratoconic eyes, Invest. Ophthalmol. Vis. Sci. 49 (2008) 5645-5652.

[35] N. López-Gil, N. Chateau, J.F. Castejón-Monchón, P. Artal, A. Benito, Correcting ocular aberrations by soft contact lenses, S. Afr. Optom. 62 (2003) 173-177.

[36] M. Chen, R. Sabesan, K. Ahmad, G. Yoon, Correcting anterior corneal aberration and variability of lens movements in keratoconic eyes with back-surface customized soft contact lenses, Opt. Lett. 32 (2007) 3203-3205.

[37] R. Sabesan, T.M. Jeong, L. Carvalho, I.G. Cox, D.R. Williams, G. Yoon, Vision improvement by correcting higher-order aberrations with customized soft contact lenses in keratoconic eyes, Opt. Lett. 32 (2007) 1000-1002.

[38] J.D. Marsack, K.E. Parker, Y. Niu, K. Pesudovs, R.A. Applegate, On-eye performance, of custom wavefrontm guided soft contact lenses in a habitual soft lenswearing keratoconic patient, J. Refract. Surg. 23 (2007) 960-964.

[39] J.D. Marsack, K.E. Parker, R.A. Applegate, Performance of wavefront-Guided soft lenses in three keratoconus subjects, Optom. Vis. Sci. 85 (2008) 1172-1178.

[40] C. Katsoulos, L. Karageorgiadis, N. Vasileiou, T. Mousafeiropoulos, G. Asimellis, Customized hydrogel contact lenses for keratoconus incorporating correction for vertical coma aberration, Ophthalmic Physiol. Opt. 29 (2009) 321-329.

[41] A. Benito, G.M. Perez, S. Mirabet, M. Vilaseca, J. Pujol, J.M. Marin, P. Artal, Objective optical assessment of tear-film quality dynamics in normal and mildly symptomatic dry eyes, J. Cataract Refract. Surg. 37 (2011) 1481-1487.

[42] R. Montes-Mico, A. Cervino, T. Ferrer-Blasco, S. Garcia-Lazaro, D. Madrid-Costa, The tear film and the optical quality of the eye, Ocul. Surf. 8 (2010) 185-192.

[43] G. Carracedo, A. Recchioni, N. Alejandre-Alba, A. Martin-Gil, L. Batres, I.J. Morote, J. Pintor, Signs and symptoms of dry eye in keratoconus patients before and after intrastromal corneal rings surgery, Curr. Eye Res. 42 (2017) 513-519.

[44] S. Ortiz-Toquero, G. Rodriguez, V. de Juan, R. Martin, Repeatability of wavefront aberration measurements with a placido-Based topographer in normal and keratoconic eyes, J. Refract. Surg. 32 (2016) 338-344.

[45] A. Jinabhai, H. Radhakrishnan, C. O'Donnell, Repeatability of ocular aberration measurements in patients with keratoconus, Ophthalmic Physiol. Opt. 31 (2011) 588-594. 